\title{
Similitude des ondulations de sable des modèles réduits et des dunes du désert
}

\section{Similitude between sand waves on small-scale models and dunes in the desert}

\author{
PAR L. FSCANDE \\ INGÉNIEUR I.E.T. \\ Professeur a la Faculté des Schences \\ Dinecteun DE r'E.N.S.E.H. DE TOULOUSJ
}

English synopsis p. 193

Dans la technique des études sur modèles réduits des ouvrages hydrauliques à fond affouil- lable, on est souvent obligé d'éliminer l'emploi de sable fin : l'utilisation de ce matériau con-

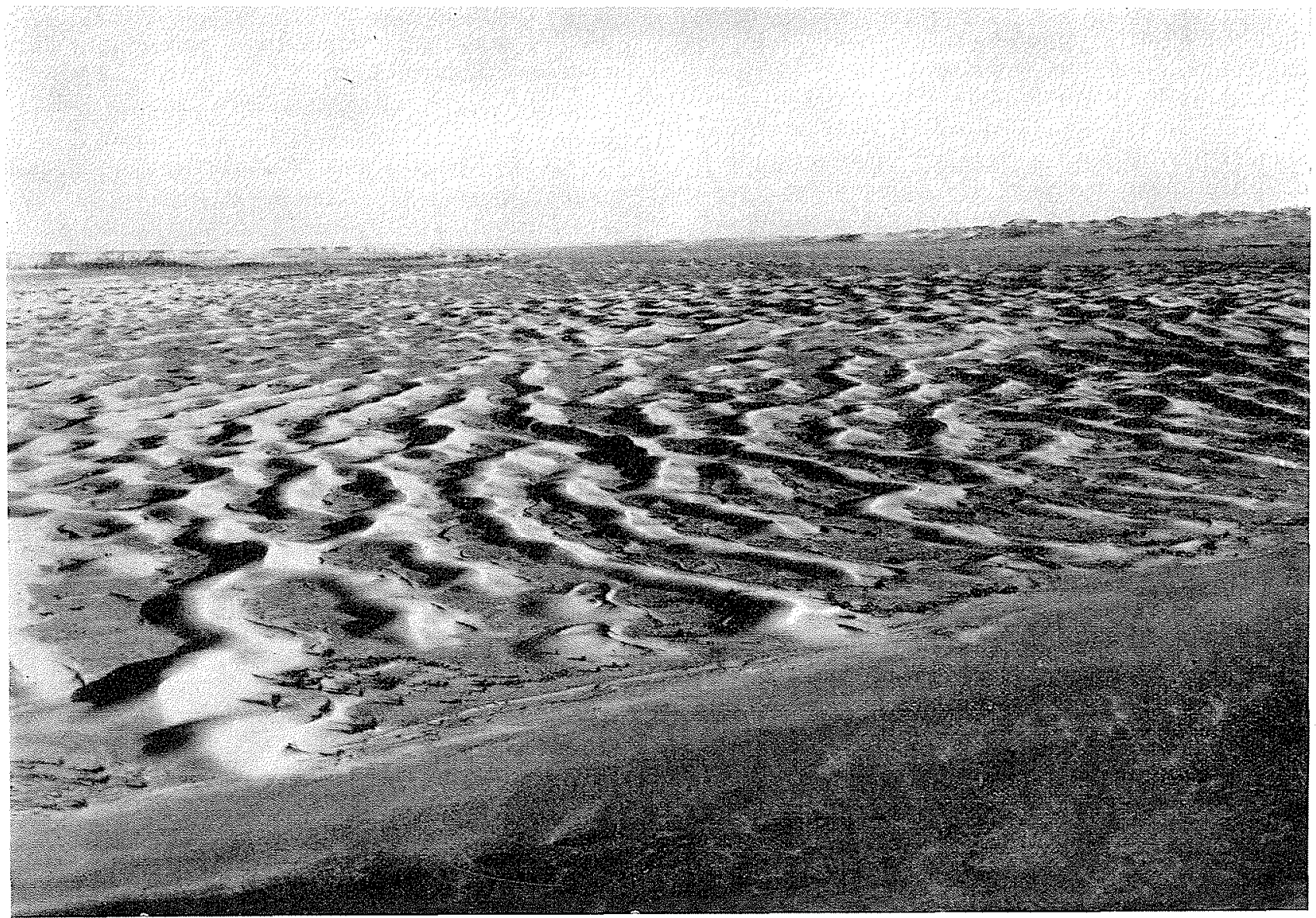

FIG. 1. 


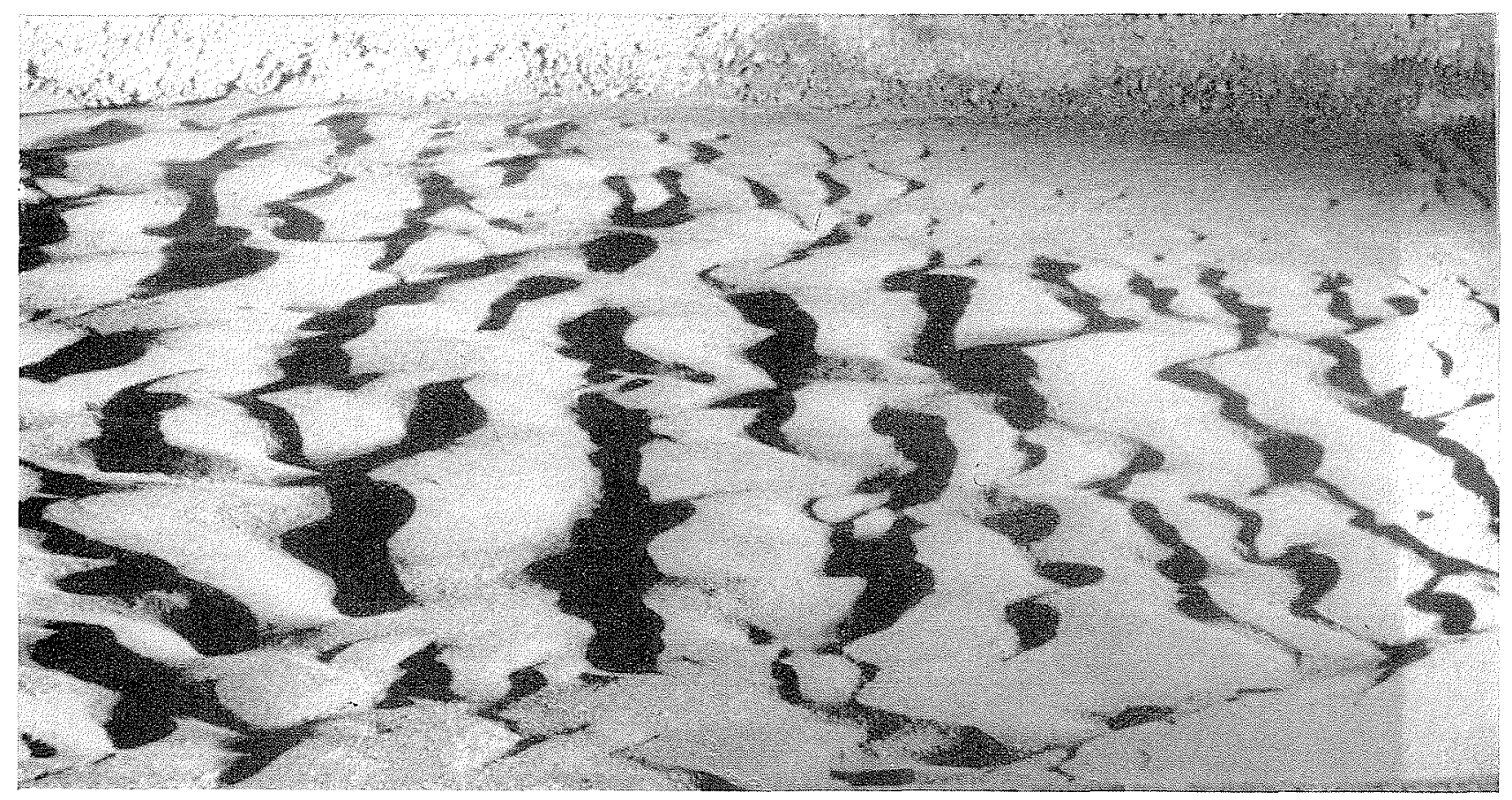

FIC. 2.

duit, en effet, à un phémomène bien connu des expérimentateurs, et consistant dans la formation, dans le lit du modèle, d'une série d'ondes de sable au tracé plus ou moins tourmenté, désignées parfois sous le nom de «Riffelbildung»; ce phénomène perturbe l'écoulement et fausse l'extrapolation à l'ourrage réel des résultats obtenus sur le modèle.

En survolant certaines régions de l'Afrique, nous avions été frappés de la similitude d'aspect vraiment curieuse existant entre les rides ainsi produites sur le fond sablonneux des modeles réduits, d'une part, et certaines configurations

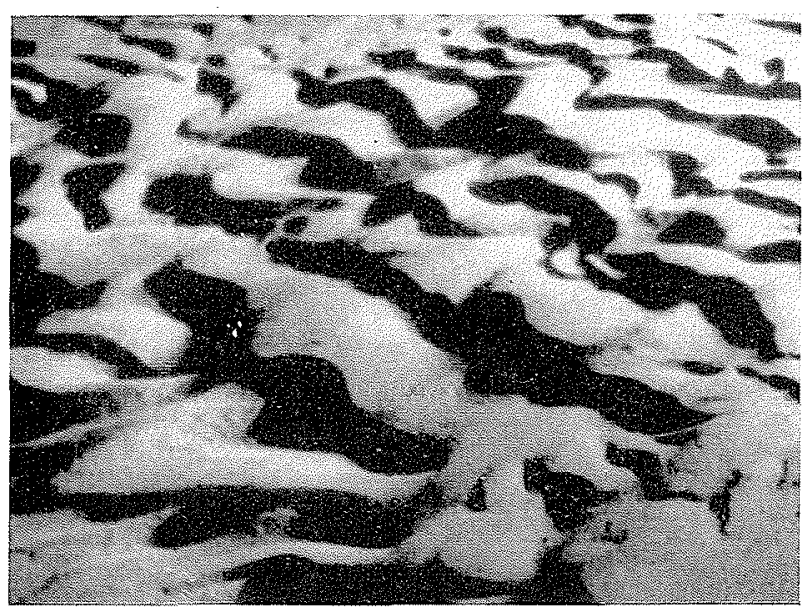

Fig. 3. des dunes de sable' engendrées par le vent dans le désert, d'autre part.

Ce souvenir s'est imposé à notre esprit, tout dernierement, lorsque nous avons eu loccasion de lire le remarquable ouvrage, Géographie aćrienne, de M. Emmanuel de Martonne, et d'observer les beaux clichés du livre Déconverte aérienne du Monde, préfacé par lui, et publié, sous la direction de M. Chombart de LaUwe, par les éditions «Horizons de France».

En particulier, la photographie de la figure n" 1, prise par M. Graule, et tirée de ce dernier ouvrage, a retenu notre attention par l'étomnante ressemblance entre le paysage de dunes qu'elle représente et les ondes de sable des modèles réduits d'ouvrages hydrauliques.

Pour préciser cette impression, nous avons réalisé une expérience particulièrement simple, dans un canal à section droite rectangulaire, de $2 \mathrm{~m}$ do largeur, dont le fond était constitué par du sable de Garonne, passé à travers un tamis pereé de trous circulaires de diamètre égal à $2,5 \mathrm{~mm}$. Nous avons fait circuler dans ce canal, pendant 1 heure 35 minutes, un débit de $121 \mathrm{l} / \mathrm{sec}$, avec un tirant d'eau moyen de l'ordre de $15 \mathrm{~cm}$; nous avons ensuite arrêté l'écoulement, asséché le modèle et observé les ondulations de sable, enserydrées par ce courant : celles-ci, dont les photographies des figures 2 et 3 reproduisent certains aspects, ressemblent à s'y méprendre à celles des dunes du désert visibles sur la figure $\mathrm{n}^{\circ} \mathbf{1}$. 


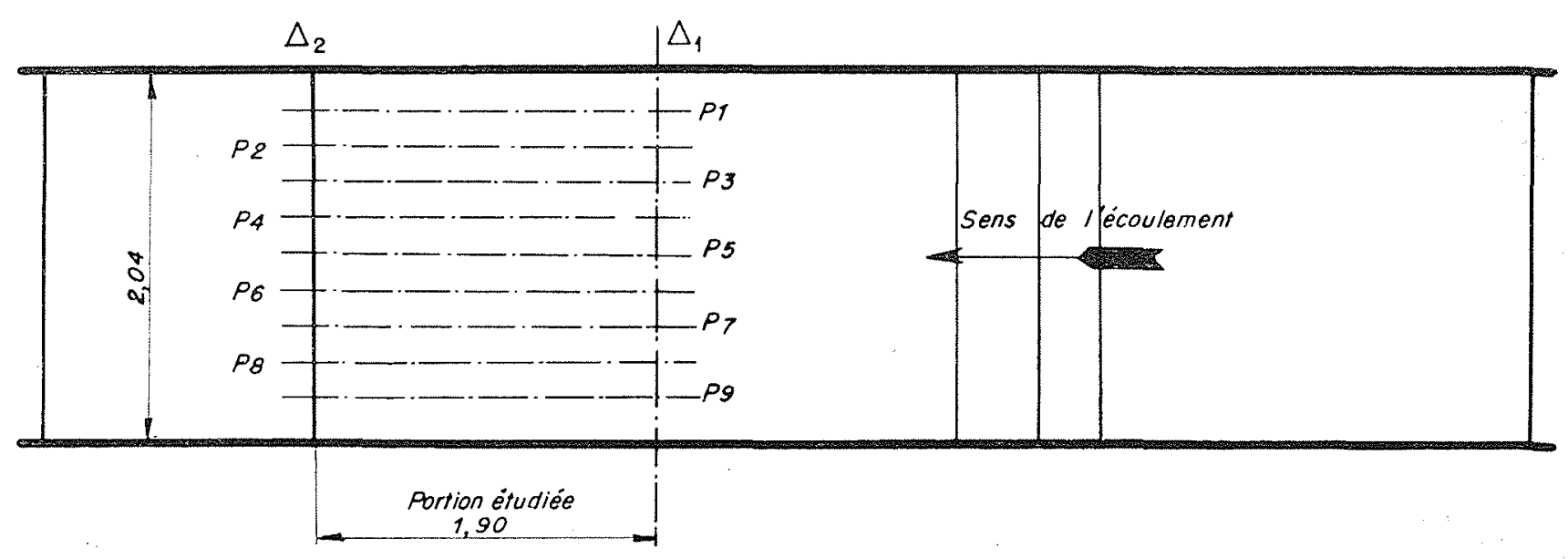

- VUE ÉLÉVATION COUPE -

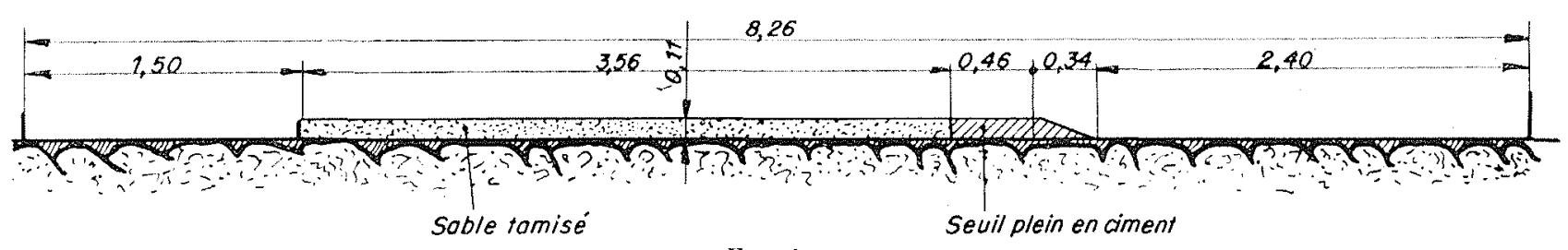

Fia. 4.

La figure $n^{\circ} 4$ donne, de facon plus précise, les dimensions du canal étudié et la position des profils verticaux parallèles à la direction générale de l'écoulement, selon lesquels le relevé du relief du fond sablonneux a été efrectuć : de creux à sommet, la distance verticale oscille en générai entre 20 et $40 \mathrm{~mm}$, comme on le voit sur le tableau $n^{\circ} 1$.

Nous avons également obtenu, sous l'action d'un courant d'air parcourant un couloir étroit, à section droite rectangulaire et à fond de sable, les ondulations, d'ailleur's moins caractéristiques, visibles sur les photographies des figures $n^{\circ 5} 5$ et 6 (sable fin de Bayonne), et 7 et 8 (sable de Garonne). Ces photographies sont prises apres enlevement de la glace constituant le plafond du tumnel.

\section{Similitude aérohydrodynamique.}

Le phínomène dont nous venons de parler correspond à une similitude acrohydrodynamique particulierement curieuse. Son interprétation demande de considérer séparément les phénomènes à deux échelles, essentiellement différentes :

1. Le mouvement d'ensemble du fluide, air ou eau, au contact de la paroi à rugosité très accentuée que constitue la surface du désert ou le fond du modèle, correspond à une échelle $\lambda$. Les vitesses et les dimensions mettent en jeu des nombres de Reynolds assez grands pour que ce

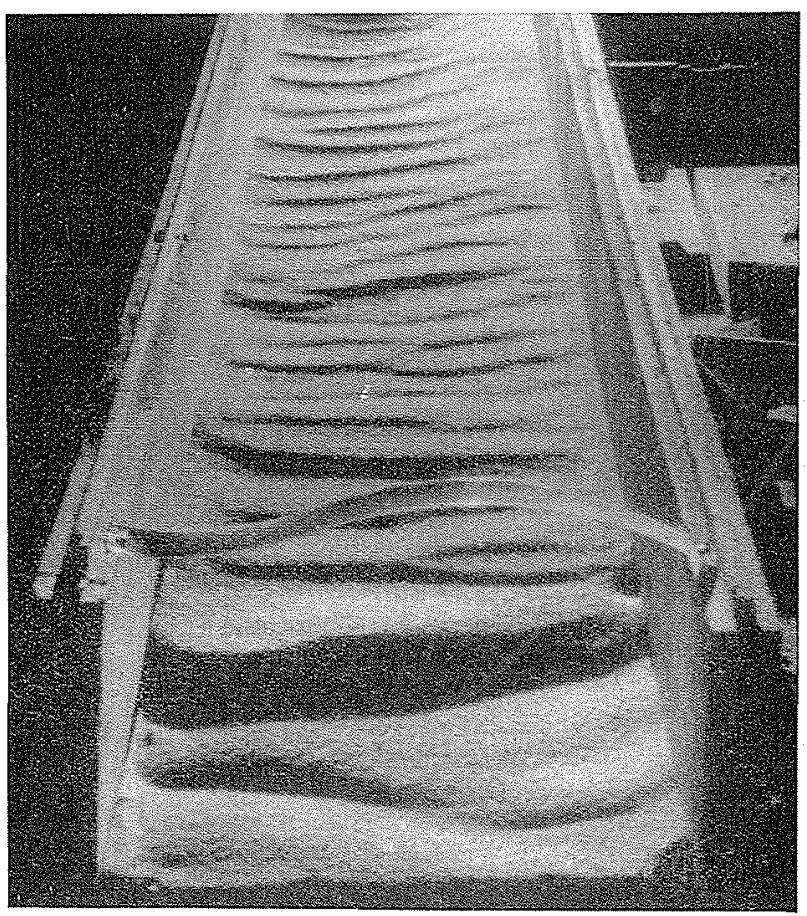

Fic, 5. 


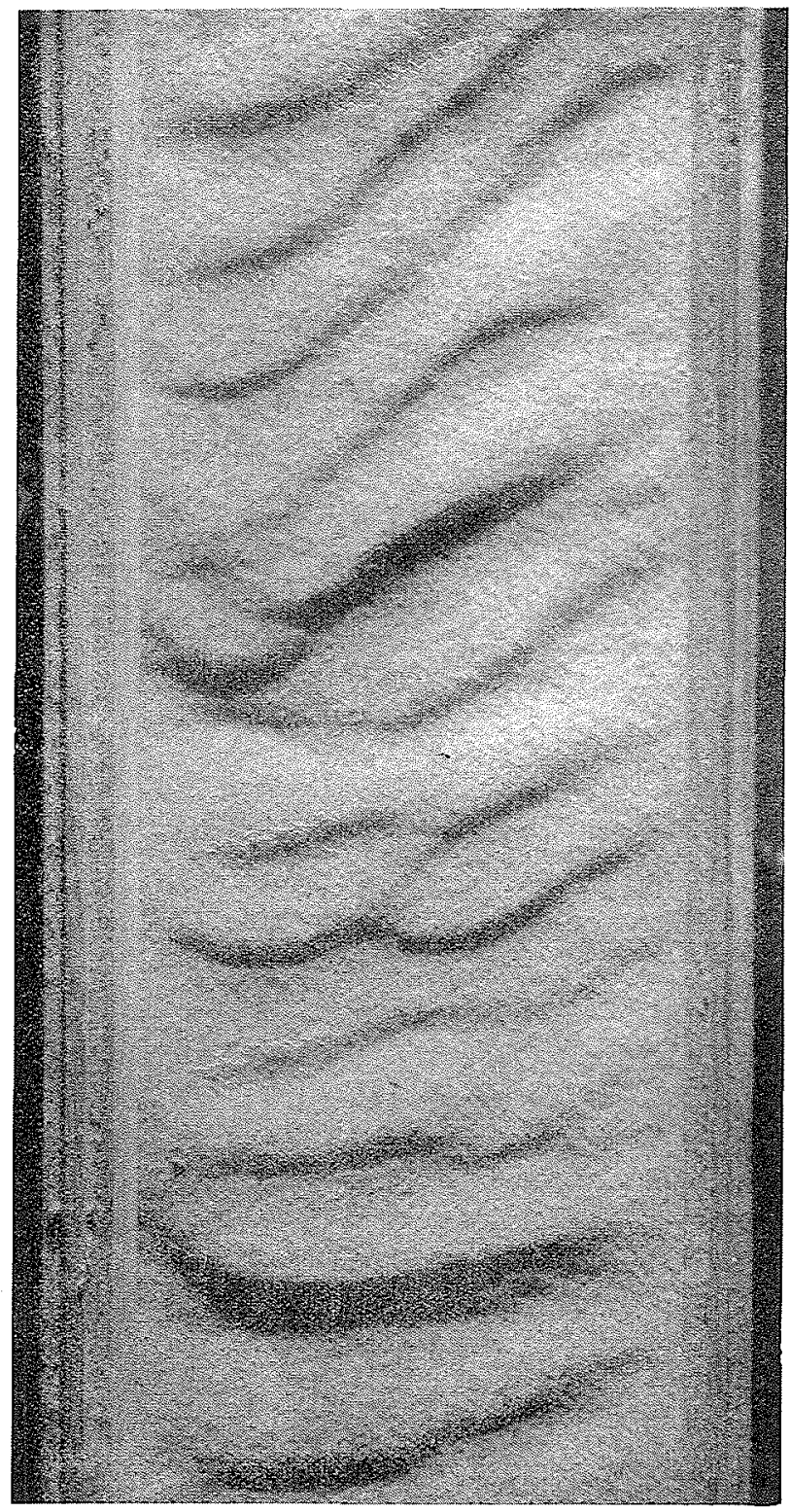

FIG. 6 .

mouvement d'ensemble ne dépende guère de la viscosité, mais, essentiellement, de la pesanteur, agissant sur les matériaux transportés; on doit done aroir, entre vitesses homologues V, la relation :

$$
-\frac{V_{2}}{V_{1}}=\sqrt{\lambda}
$$

l'indice 2 correspondant au mouvement de l'air dans le désert, l’indice 1 à celui de l'eau dans le modèle réduit.

$2^{\circ}$ A l'échelle $\lambda$ des dimensions linéaires moyennes $D$ des grains transportés dans les deux mouvements comparés, la pesanteur el la viseo-

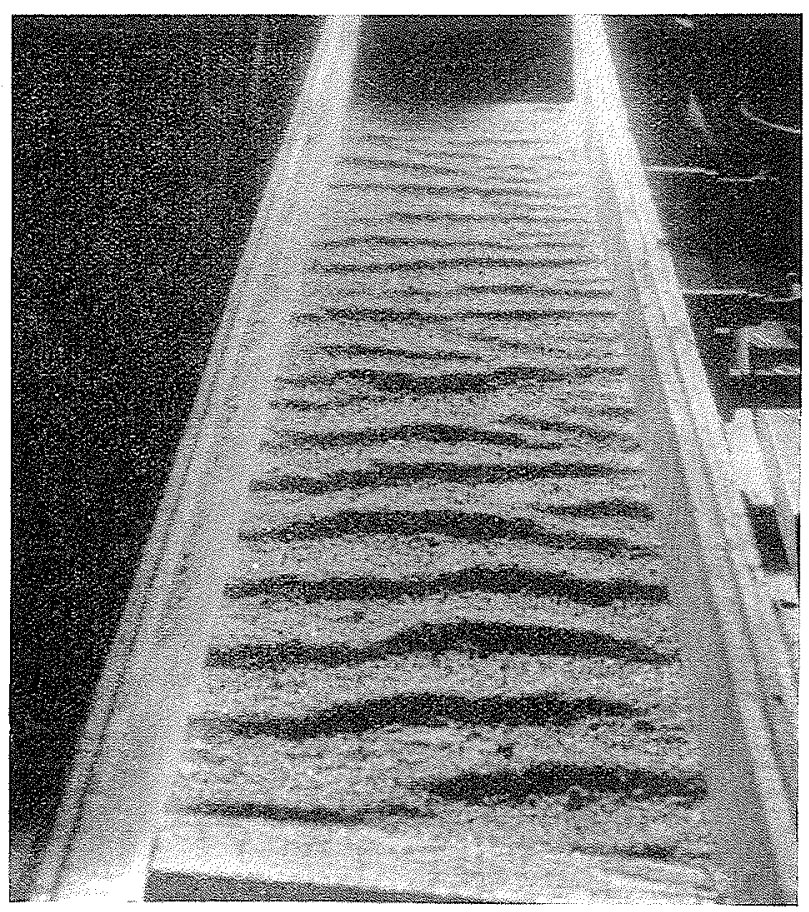

Fig. 7.

sité interviennent simultanément et l'on doit avoir :

$$
\begin{aligned}
& \frac{V_{2} D_{2}}{y_{2}}=\frac{V_{1} D_{1}}{v_{1}} \text { ou } \frac{V_{2}}{V_{1}}=\frac{1}{\lambda} \frac{y_{2}}{y_{1}} \\
& \frac{\rho_{2} \mathrm{D}_{2}{ }^{2} \mathrm{~V}_{2}{ }^{2}}{\rho_{1} \mathrm{D}_{1}{ }^{2} \mathrm{~V}_{1}{ }^{2}}=\frac{d_{2} \mathrm{D}_{2}{ }^{3}}{d_{1} \mathrm{D}_{1}{ }^{3}} \text { ou } \frac{\mathrm{V}_{2}}{\mathrm{~V}_{1}}=\sqrt{\lambda \frac{d_{2} \rho_{1}}{d_{1} \rho_{2}}(3)}
\end{aligned}
$$

en désignant par , le coefficient cinématique de viscosité du fluide, par o sa densité et par $d$ la densité dans le fluide correspondant du matériau transporté (poids dans l'air ou dans l'eau).

On tire des conditions (1), (2), (3) les relalions suivantes:

$$
\begin{aligned}
& \lambda=\sqrt[3]{\frac{d_{1}}{d_{2}}, \frac{\rho_{2}}{\rho_{1}}\left(\frac{\nu_{2}}{\nu_{1}}\right)^{2}}, \frac{V_{2}}{V_{1}}=\sqrt[3]{\frac{d_{2}}{d_{1}}, \frac{\rho_{2}}{\rho_{1}}, \frac{\nu_{2}}{\nu_{1}}}, \\
& x^{\prime}=\left(\frac{V_{2}}{V_{1}}\right)^{2}=\left[\frac{d_{2}}{d_{1}} \frac{\rho_{1}}{\rho_{2}}, \frac{\nu_{2}}{\nu_{1}}\right]^{2 / 3} \text {. }
\end{aligned}
$$

Ainsi, dans le cas de l'expérience sur modèle citée précédemment, si l'on admet que :

$$
\begin{gathered}
\frac{d_{2}}{d_{1}}=\frac{2,4}{1,4}=1,715 \quad \frac{\rho_{2}}{\varrho_{1}}=\frac{1}{800} \\
\frac{y_{2}}{\nu_{1}}=15
\end{gathered}
$$

on $\mathrm{a}$ :

$$
\lambda=0,547 \quad \frac{V_{2}}{V_{1}}=27,4 \quad \lambda^{\prime}=751
$$




\section{TABLEAU N"}

Nota. - Les deux chiffres donnés pour chaque point indiquent, le premier, la distance à $\Delta_{1}$, le deuxième, l'allitude en centimètres, positive ou négative, par rapport au plan horizontal constituant la surface du fond initial.

\begin{tabular}{|c|c|c|c|c|c|c|c|c|c|c|c|c|c|c|c|c|c|}
\hline \multicolumn{9}{|c|}{ NUMÉROS DES PROFILS } & \multicolumn{9}{|c|}{ NUMÉROS DES PROFILS } \\
\hline 1 & 2 & 3 & 4 & 5 & 6 & 7 & 8 & 9 & 1 & 2 & 3 & 4 & 5 & 6 & 7 & 8 & 9 \\
\hline \multicolumn{9}{|c|}{ Distances a la RIVE DROITE } & \multicolumn{9}{|c|}{ Distances a la RIVE DROITE } \\
\hline 20 & 40 & 60 & 80 & 100 & 120 & 140 & 160 & 180 & 20 & 40 & 60 & 80 & 100 & 120 & 140 & 160 & 180 \\
\hline 0 & 0 & 0 & 0 & 0 & 0 & 0 & 0 & 0 & 154 & 148 & 1131 & 144 & 134 & 95 & 120 & 190 & \\
\hline$=0,45$ & $-0, \overline{45}$ & $-0,10$ & $-0,25$ & $-1,10$ & $=0,25$ & $-0,05$ & $-1,101$ & $+2,25$ & $-0,90$ & $=0,10$ & $-0,75$ & $+1,00$ & $-0,05$ & $-1,75$ & $+0,15$ & 0,00 & \\
\hline & & & & & & & & & & & & & & & & & \\
\hline 301 & 30 & 25 & 34 & 12 & $19 i$ & 30 & 10 & 19 & 160 & 150 & 1281 & 146 & 137 & 961 & 122 & & \\
\hline$-0,70$ & $-0,25$ & $+0,10$ & $+-0,85$ & $-0,65$ & $|-0,10|$ & $-1,15$ & $-4,65$ & $+2,05$ & $=0,65$ & $-0,55$ & $|-0,15|$ & $-0,25$ & $-0,65$ & $-2,15$ & $-1,05 \mid$ & & \\
\hline & & & & & & & & & & & & & & & & & \\
\hline 60 & 60 & 27 & 36 & 14 & 201 & 37 & 73 & 22 & 165 & 162 & $132 \mid$ & 163 & 143 & 102 & $140 \mid$ & & \\
\hline$+0,05$ & $+0,05$ & $-0,751$ & $|-0,45|$ & $-1,15$ & $-0,65 \mid$ & $-2,15$ & $+0,95$ & $+0,05$ & $=0,75$ & $+0,35$ & $|-2,40|$ & $+1,05$ & $-0,95$ & $-1,20$ & $-0,90$ & & \\
\hline & & & & & & & & & $=1$ & & & & & & & & \\
\hline 81 & 84 & 41 & 43 & 28 & 29 & 42 & 80 & 43 & 170 & 164 & 139 & 175 & 154 & 103 & 144 & & \\
\hline$=0,15$ & $+0,25$ & $-0,20)$ & $-0,35$ & $-0,25$ & $-0,25 \mid$ & $-0,25$ & $-1,20$ & $+1,25$ & $-0,20$ & $-0,55$ & $-0,25$ & $+0,55$ & $+0,30$ & $|-1,45|$ & $-2,45$ & & \\
\hline & & & & & & & & & & & & & & & & & \\
\hline 83 & 86 & 43 & 45 & 30 & 301 & 45 & 100 & 69 & 175 & 173 & 142 & 180 & 155 & 115 & 148 & & \\
\hline$=0,55$ & $=0,15$ & $-0,95$ & $|-0,95|$ & $-1,35$ & $|-0,85|$ & $-1,35$ & $+0,25$ & $+1,45$ & $-2,35$ & $-0,85$ & $-1,50$ & $-0,15$ & $-0,45$ & $-0,05$ & $|-2,10|$ & & \\
\hline & & & & & & & & & & & & & & & & & \\
\hline 88 & 88 & 50 & 60 & 50 & 46 & 48 & 105 & 110 & 180 & 175 & 157 & 186 & 165 & 120 & 158 & & \\
\hline$-0,25$ & $+0,05$ & $=0, \overline{65}$ & $+1,25$ & $+0,45$ & $|-0,45|$ & $-1,50$ & $-0,55 \mid$ & $-0,15$ & $-1,15$ & $-1,90$ & $+0,85$ & $=0,60$ & $-0,25$ & $|-2,40|$ & $+0,75$ & & \\
\hline & & & & & & & & & & & & & & 1 & & & \\
\hline 98 & 93 & 52 & 67 & 52 & 47 & 55 & 110 & 115 & 185 & 182 & 159 & 190 & 167 & 123 & 161 & & \\
\hline $\pm 0,05$ & $+0,15$ & $-1,60$ & $-0,75$ & $-0,45$ & $|-|, 15 \mid$ & $-0,25$ & $-0,45 \mid$ & $-0,75$ & $=0,35$ & $=0,75$ & $-0,25$ & 0,00 & $1-1,40$ & $|-2,85|$ & $-1-0,05$ & & \\
\hline & & & & & & & & & & & & & & & & & \\
\hline 99 & 95 & 59 & 77 & 72 & 59 & 57 & 125 & 150 & 190 & 187 & $180 \mid$ & & 183 & 128 & 164 & & \\
\hline$-0,45$ & $-0,40$ & 0,00 & $-0,85$ & $+0,85$ & $|-0,05|$ & $-1,00$ & $+0,75$ & $-0,45$ & 0,00 & $-0,40$ & $-0,45 \mid$ & & $-0,05 \mid$ & $|-1,95|$ & 0,00 & & \\
\hline & & & & & & & & & & & & & $i$ & & & & \\
\hline 104 & 104 & 61 & 80 & 76 & 60 & 66 & 134 & 190 & & 190 & 184 & & 184 & 148 & 170 & & \\
\hline$-0,15$ & $-0,30$ & $-1,25$ & $-2,25$ & $-0,65$ & $-0,65 \mid$ & $+0,25$ & $+0,25$ & $-0,05$ & & 0,00 & $|-2,75|$ & & $|-0,75|$ & $|+1,05|$ & $-0,55$ & & \\
\hline & & & & & & & & & & & & & & & & & \\
\hline 110 & 105 & 76 & 84 & 86 & 67 & 67 & 153 & & & & 190 & & 190 & 149 & 190 & & \\
\hline $\pm 0,40$ & $=0,75$ & $+0,15$ & $-1,45$ & $-0,05$ & $|-0,15|$ & $-0,55$ & $-0,75$ & & & & $|-0,65|$ & & $1+0,05$ & $1+0,20$ & $+0,95$ & & \\
\hline & & & & & & & & & & & & & & & & & \\
\hline 116 & 109 & 79 & 95 & 90 & 70 & 83 & 154 & & & & & & & 170 & & & \\
\hline$+0,15$ & $=1,10$ & $-1,25$ & $+-0,75$ & $-1,45$ & $|-1,00|$ & $+0,45$ & $-1,70$ & & & & & & & $|+0,65|$ & & & \\
\hline & & & & & & & & & & & & & & & & & \\
\hline 118 & 111 & 85 & 96 & 100 & 79 & 85 & 164 & & & & & & & 172 & & & \\
\hline$-0,40$ & $-1,90$ & $1+0,35$ & $-0,10$ & $1+1,25$ & $-0,25$ & $-0,35$ & $-1,50$ & & & & & & & $-0,15$ & & & \\
\hline & & & & & & & & & & & & & & 1 & & & \\
\hline 130 & 114 & 91 & 115 & 101 & 81 & 93 & 165 & & & & & & & 184 & & & \\
\hline$=0,60$ & -0.55 & $+0,75$ & $+0,35$ & $+0,35$ & $-1,75$ & $+0,80$ & $-2,60$ & & & & & & & $+0,25$ & & & \\
\hline & & & & & & & & & & & & & & & & & \\
\hline 142 & 131 & 92 & 119 & 117 & 90 & 95 & 174 & & & & & & & 190 & & & \\
\hline$=0,05$ & $+0,05$ & 0,00 & $-1,50$ & $+0,25$ & $|-1,85|$ & $-0,55$ & $|-1,10|$ & & & & 1 & & & 0,00 & & & \\
\hline & & & & & & & & & & & - & & & & & & \\
\hline 1431 & 133 & 110 & 130 & 120 & 93 & 106 & 185 & & & & & & & & & & \\
\hline$-0,95$ & $-1,10$ & $+1,25$ & $-0,75$ & $-2,25$ & $-2,40$ & $-0,25$ & $+0,55$ & & & & & & & & & & \\
\hline
\end{tabular}


Compte tenu des données du modèle hydraulique (sable passant à l'anneau de $2,5 \mathrm{~mm}$, ondulations de 2 a $4 \mathrm{~cm}$, vilesse moyenne du courant $40 \mathrm{~cm} / \mathrm{sec}$.), les dunes semblables, d'un relief de 15 à 30 mètres, seraient obtenues pour un vent de $40 \mathrm{~km}$ à l'heure, avec du sable passant à lanneau de $1,4 \mathrm{~mm}$.

Notons que, lors de l'expérience sur le modile réduit hydraulique, on pouvait faire apparaitre les ondulations, d'ailleurs plus petites, pour des vitesses notablement plus fables que $40 \mathrm{~cm} / \mathrm{sec}$.

Pour opérer de façon plus précise, il conviendrait de partir des données caractérisant une zone de dunes désertiques assez bien définies $\left(V_{2}, D_{2}, d_{2}\right)$, d'en déduire, par les relations cidessus, les données à faire intervenir dans le modèle hydraulique, et d'observer les caractéristiques des ondulations obtenues, dans ces conditions, sur son fond affouillable.

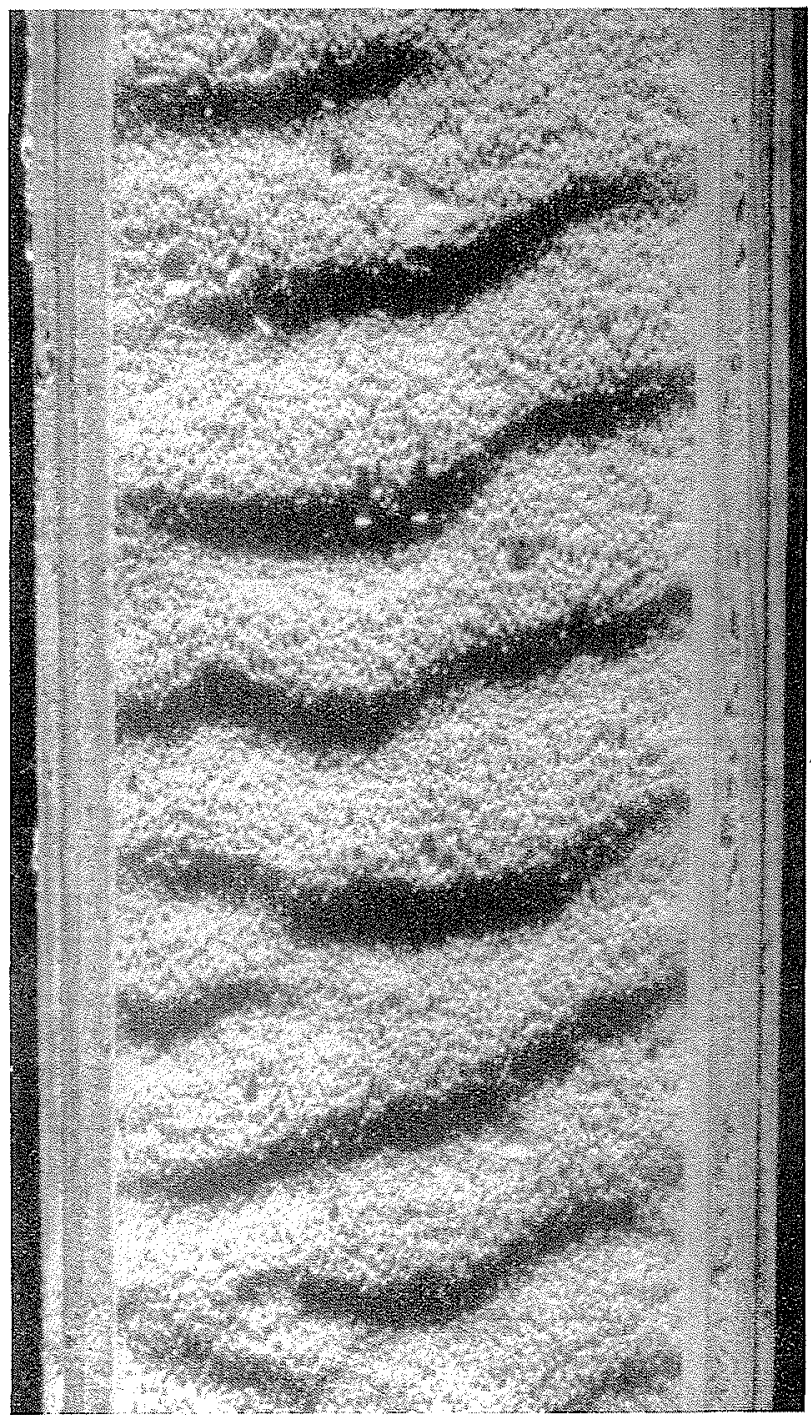

Fici.8. 